\title{
Towards a Multi-modal Atlas for Neurosurgical Planning
}

\author{
M. Mallar Chakravarty ${ }^{1}$, Abbas F. Sadikot ${ }^{1,2}$, Sanjay Mongia ${ }^{2}$, \\ Gilles Bertrand ${ }^{1,2}$, and D. Louis Collins ${ }^{1}$ \\ ${ }^{1}$ McConnell Brain Imaging Centre \\ ${ }^{2}$ Department of Neurosurgery \\ Montreal Neurological Institute \\ 3801 University Street, Montreal, Quebec \\ Canada, H3A 2B4
}

\begin{abstract}
Digital brain atlases can be used in conjuction with magnetic resonance imaging (MRI) and computed tomography (CT) for planning and guidance during neurosurgery. Digital atlases are advantageous since they can be warped nonlinearly to fit each patient's unique anatomy. Functional neurosurgery with implantation of deep brain stimulating (DBS) electrodes requires accurate targeting, and has become a popular surgical technique in Parkinsonian patients. In this paper, we present a method for integrating postoperative data from subthalamic (STN) DBS implantation into an antomical atlas of the basal ganglia and thalamus. The method estimates electrode position from post-operative magnetic resonance imaging (MRI) data. These electrodes are then warped back into the atlas space and are modelled in three dimensions. The average of these models is then taken to show the region where the majority of STN DBS electrodes were implanted. The group with more favorable post-operative results was separated from the group which responded to the STN DBS implantation procedure less favourably to create a probablisitic distribution of DBS in the STN electrodes.
\end{abstract}

\section{Introduction}

Deep brain stimulation (DBS) has become a popular method to treat movement disorders such as Parkinson's Disease [2 8 15 20]. Despite current improvements in imaging techniques [20], magnetic resonance imaging alone cannot suggest the final location for surgical targets. For intra-operative guidance, neurosurgeons will typically use a combination of pre-operative imaging, atlas-integration, stimulation of the internal capsule and subcortical nuclei, as well as electrophysiological recording in order to properly localize these surgical targets. Originally, print atlases were used to guide these surgical procedures 1821. Advances in medical image processing techniques have enabled the use of digital atlases representing the subcortical anatomy to be warped to match the individual patient anatomy and have been used to aid the visualization of surgical targets 1145919 . The use of multi-modal atlases has grown in popularity to aid in 
target localization [910121316]. The integration of functional recording (such as electrode stimulation recordings and electrophysiological recordings of the internal capsule and subcortical nuclei 9,11,12,13]) and the post-operative position of active DBS electrodes in the subthalamic nucleus using linear and nonlinear mappings 910,16 have been used to create a new class of atlases called the probabilistic functional atlas.

In this paper we propose a new technique to create a probabilistic functional atlas based on an estimation of electrode position in post-operative data from Parkinson's patients who underwent STN DBS implantation. In the creation of our probabilistic functional atlas each electrode was modelled as a three dimensional structure based on the geometry of each electrode in the DBS. All electrodes are then warped towards a common template using nonlinear atlasto-patient warping techniques based on [5].

The functional information has been added to an anatomical atlas of the basal ganglia and thalamus derived from a high-resolution set of serial histology 4]. This atlas contains 105 structures and has been nonlinearly warped to fit a high resolution MRI template known as the Colin27 MRI average [14. The anatomical atlas can be seen in Fig. 1] The accuracy of this atlas to template warp was validated in [5].

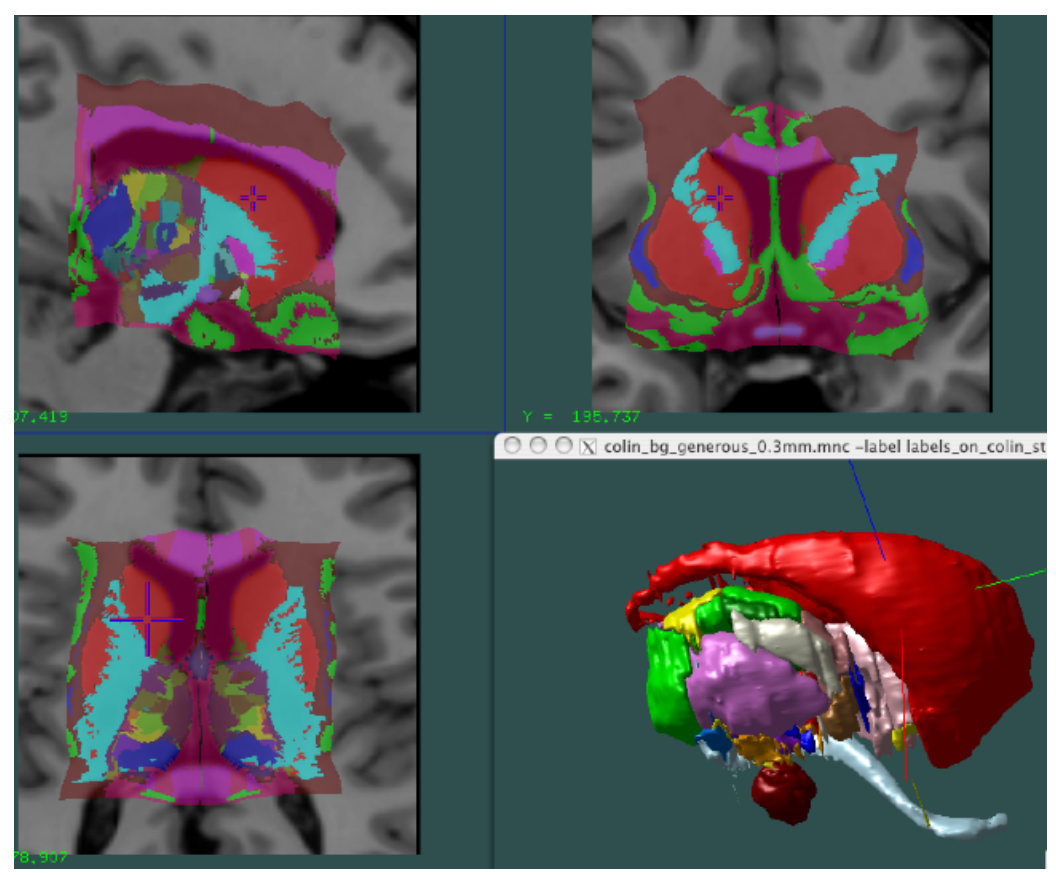

Fig. 1. First three panels: Voxel-label atlas warped to fit the Colin27 MRI average. Bottom Right: 3D geometric atlas. 
In this paper we will explain the methods used to create the probabilistic functional atlas (PFA) using postoperative data in Section 2, The results of the creation of the PFA are given in Section 3.

\section{Probabilistic Functional Atlas Creation Techniques}

The electrode model used in this paper is based on the actual geometry of the electrodes in the DBS. Details of the 3D model are given in Section 2.1. The estimation of the electrode position in postoperative data from patients who underwent STN DBS electrode implantation is described in Section 2.2. The method used to warp the electrode position in a final common atlas space is described in Section 2.3. Once all DBS electrodes are warped to the atlas, the probabilistic atlas is created (see Section 2.4). The electrode position was estimated on post-operative MRI data from 22 patients who underwent subthalamic STN DBS implantation.

\subsection{D Electrode Model}

Each electrode in the dataset is modelled as a cylinder in a $3 \times 3 \times 3 \mathrm{~cm}$ binary volume on a grid with a $0.1 \mathrm{~mm}$ isotropic voxel-to-voxel spacing. All voxels considered to belong to the electrode are set to an intensity value of 1 where all voxels outside of the electrode are set to 0 . The diameter of cylinder is was 1.3 $\mathrm{mm}$ and the height was $1.5 \mathrm{~mm}$ (based on the actual geometry of the electrodes). A chamfer distance map 3 is then estimated so that region around the electrode can be modelled as a decaying electric field using a simple $1 / r^{2}$ model. This is an approximation of the influence of the electrode and will be discussed further in the conclusions presented in Section 4. This model is then blurred with a $2 \mathrm{~mm}$ isotropic Gaussian blur to account for the uncertainty inherent in the model. A slice thrugh the volume of the initial cylinder, the $1 / r^{2}$ and the blurred $1 / r^{2}$ maps can be seen in Fig. 2.
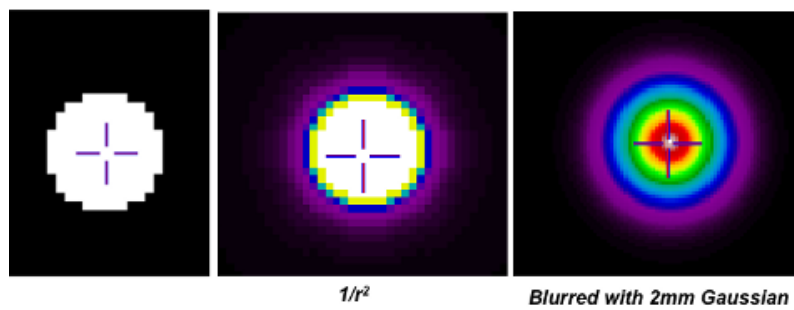

Fig. 2. Electrode model. From left to Right: A top view of the initial binary electrode model, a view of the modeled $1 / r^{2}$ electric field around the electrode, and the electric field blurred with a $2 \mathrm{~mm}$ isotropic Gaussian kernel. 


\subsection{Electrode Position Estimation}

We assume that that any MRI artefact caused by the DBS lead is homogeneous in all three dimensions. For each subject, two points were manually identified. The first point, $T$, identified the top of the tract of the STN DBS and the second, $B$ identified the bottom. The midpoint, $M$ of the line segment defined by $\overline{B T}$ was calculated. A unit row vector $\overrightarrow{\boldsymbol{u}}$ was defined using $\overline{B T}$ in the direction $T$. Using the $M$ and $\overrightarrow{\boldsymbol{u}}$ the position of the four electrodes in each DBS lead are estimated using simple vector algebra: $E=P \overrightarrow{\boldsymbol{u}}+M$, where $E=\left[E_{3}, E_{2}, E_{1}, E_{0}\right]^{T}$ is a column vector vector of the final electrode position, and $P=\left[P_{3}, P_{2}, P_{1}, P_{0}\right]=$ $[2.25,0.75,-0.75,-2.25]$ is a column vector of magnitudes of the distance (in $\mathrm{mm}$ ) of each electrode away from the $M$, based on the actual geometry of the DBS. The result of the electrode estimation can be seen in Fig. 3.
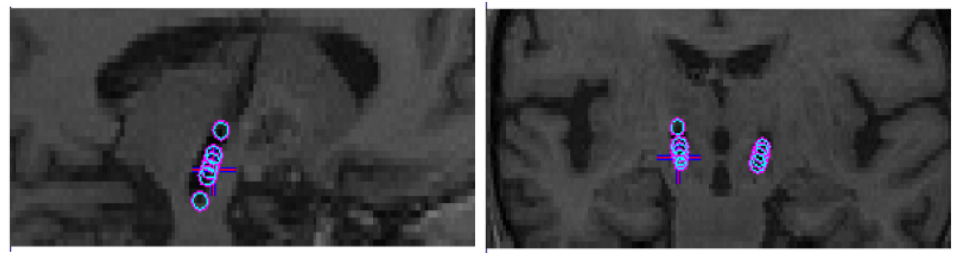

Fig. 3. Electrode Estimation. From left to Right: Sagittal and coronal views of the DBS electrode position estimation procedure. Top and bottom landmarks of the tract are clearly visible in sagittal view. Note that DBS electrodes are not necessarily visible in the coronal or sagittal planes and that the electrode positions are projected to these slices.

\subsection{Nonlinear Patient-to-Atlas Warping}

Once the electrode positions have been estimated on the post-operative data they need to be warped into a common atlas space. The post-operative MRI data is first linearly warped using a rigid body transformation (3 translations and 3 rotations) 7 to the pre-operative MRI for each subject. The nonlinear patient-toatlas transformation is then estimated from the pre-operative data to the atlas. This is done to eliminate the effects of artefacts caused by DBS electrodes in the post-operative data on the nonlinear transformation procedure described next.

The nonlinear warping technique uses a modified version of the ANIMAL algorithm [6]. ANIMAL is an iterative algorithm which estimates a 3D deformation field which matches a source volume to the target volume. The transformation estimation consists of two steps: calculating a deformation at each node which will maximize the local similarity measure, and the second is a smoothing step to ensure that a continuous deformation field has been estimated. For further details on ANIMAL's parameters, the reader is referred to 46 6[17. In this implementation, similar to that in [5], we do not use blurred data, as we are estimating a high resolution deformation field. A mask in the region of subcortical nuclei is used in both the source and target data. Each nonlinear transformation estimated maximizes the similarity between the template and patient volume. The 
final nonlinear transformation is estimated in three steps, larger deformations are estimated first and become the input transformation when estimating more refined deformations. The details of the ANIMAL parameters used at each step are shown in Table 1. For each step in the transformation estimation, the same weight, stiffness, and similarity values are used $(1,1$, and 0.3 respectively, as determined in the optimization done by Robbins et al. [17]), and use the crosscorrelation objective function.

Table 1. ANIMAL parameters used for warping patient MRI data to the Colin27 template

\begin{tabular}{|c||c|c|c|c|}
\hline Step & Step Size (mm) & Sub Lattice Diameter (mm) & Sub Lattice & Iterations \\
\hline \hline 1 & 4 & 8 & 8 & 15 \\
\hline 2 & 2 & 6 & 8 & 15 \\
\hline 3 & 1 & 6 & 6 & 15 \\
\hline
\end{tabular}

\subsection{Final Functional Atlas Creation}

Using the final concatenated patient-to-atlas transformation, each electrode is warped into the atlas space. In the atlas space the roll, pitch, and yaw angle of each electrode in each DBS lead is estimated by averaging two vectors defined the current electrode and the electrode above and the electrode below. The resulting rotation matrix is applied to the active electrode models. Once all the electrode models of all the active electrodes are in the atlas space they are averaged to create a PFA. Active electrodes are defined by the neurosurgical/neurologist team caring for the patient. The total group was subdivided into two groups using preoperative versus postoperative UPDRS motor score improvement. The two groups were defined as those subjects that showed greater than $30 \%$ and $50 \%$ improvement.

\section{Results of Atlas Creation}

The results of the final multi-modal (anatomical and PFA) atlas as are shown in Fig. 4. The figure is organized as follows. On the left, 4 panels are shown. From left to right on the top panel, the anatomical atlas in the region of the STN is shown and the average of all active electrodes on all subjects is shown. Looking at the bottom panel, from left to right, we see the groups subdivided into those patients who had greater than $30 \%$ improvement (17 subjects) and those who had greater than $50 \%$ improvement (11 subjects). On the right we see the 3D geometric atlas defined in 4 in the region of the subthalamic nucleus. The blue wire-frame structure is the STN, and red surface is an isosurface defined from the PFA of patients showing greater that $50 \%$ improvement.

Using this type of volumetric data allows increased visualization of the STN area. In the bottom panels we see an expected narrowing of the probabilistic cluster which has been defined. In addition we see that this cluster approaches 

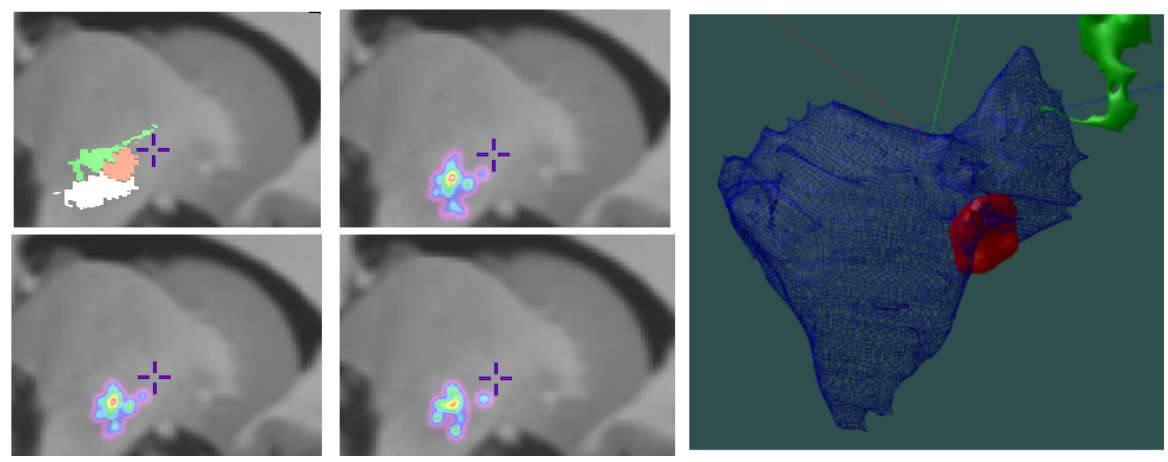

Fig. 4. First four panels left, left to right, top to bottom: The atlas definition of the Zona Incerta (ZI, in green), STN (in orange), and the substantia nigra (in white) warped to the Colin27 MRI template. PFA created from all the active electrodes, PFA created from all patients who had a $30 \%$ postoperative improvement in UPDRS, and PFA created from all patients who had $50 \%$ improvement in postoperative UPDRS. Right: Sagittal view of 3D geometric atlas of the STN in blue, and region of highest probability taken from average of patients who had a $50 \%$ improvement in UPDRS (defined as volume in red). Part of the ZI can be seen in green.

the structural definition of the STN on the anatomical atlas. The 3D geometric view of this data is also very useful for anatomical planning, as it gives an $a$ priori volumetric region for surgical targeting in the STN.

\section{Conclusions and Future Work}

In this paper we have demonstrated a method for the creation of a multi-modal atlas using a combination of anatomical and functional data. The PFA creation is based on an estimation of the final electrode position of STN DBS electrodes in postoperative MRI data. These final electrode locations on the atlas are estimated by matching the postoperative patient data to preoperative patient data with a rigid body transform. A nonlinear transformation matching the preoperative data to the atlas template is then estimated. The concatenated postoperative to template transformation is then used to transform the electrode positions to the atlas template. In the atlas template all electrodes are represented by a volumetric model. All active electrodes are averaged together and then grouped with respect the patient's response to the surgical procedure postoperative. The results demonstrate a strong relationship between the location of the group averages with the location of the STN on the anatomical atlas. In addition this information can be used intraoperatively in three dimensions in order to aid in targeting during STN DBS implantation surgeries.

Future work will include the addition of active electrode data, as well as the results of intraoperative stimulations and electrophysiological recordings in the STN region. The combination of this data will provide a powerful anatomical and functional visualization tool for preoperative and intraoperative use. The 
electrode model must be further explored to better account for tissue conductivity, as well as frequency and voltage setting of each STN DBS electrode. However it has been shown in this paper that the proposed model provides a reasonable approximation of the STN DBS and the surrounding field.

\section{References}

1. E. Bardinet, D. Dormont, G. Malandain, M. Bhattacharjee, B. Pidoux, C. Saleh, P. Cornu, N. Ayache, Y. Agid, and J. Yelnik. Retrospective cross-evaluation of an histological and deformable $3 \mathrm{~d}$ atlas of the basal ganglia on series on parkinsonian patients treated by deep brain stimulation. In Seventh International Conference on Medical Image Computing and Computer Assisted Intervention MICCAI 2005, volume 2 of Lecture Notes in Computer Science, pages 385-393, Palm Springs, USA, October 2005. Springer.

2. A.L. Benabid, A. Koudsie, A. Benazzouz, J.F. Le Bas, and P. Pollak. Imaging of subthalamic nucleus and ventralis intermedius of the thalamus. Movement Disorders, 17:S123-S129, 2002.

3. G. Borgefors. Distance Transformations in Arbitrary Dimensions. Computer Vision, Graphics, and Image Processing, 27:321-345, 1984.

4. M.M. Chakravarty, G. Bertrand, C.P. Hodge, A.F. Sadikot, and Collins D.L. The creation of a brain atlas for image guided neurosurgery using serial histological data. NeuroImage, 30(2):359-376, 2006.

5. M.M. Chakravarty, A.F. Sadikot, J. Germann, G. Bertrand, and D.L. Collins. Anatomical and electrophysiological validation of an atlas for neurosurgical planning. In Seventh International Conference on Medical Image Computing and Computer Assisted Intervention MICCAI 2005, volume 2 of Lecture Notes in Computer Science, pages 394-401, Palm Springs, USA, October 2005. Springer.

6. D.L. Collins and A.C. Evans. ANIMAL: validation and application of non-linear registration-based segmentation. International Journal of Pattern Recognition and Artificial Intelligence, pages 1271-1294, December 1997.

7. D.L. Collins, P. Neelin, T.M. Peters, and A.C. Evans. Automatic 3D Intersubject Registration of MR Volumetric Data in Standardized Talairach Space. J. of Computer Assisted Tomography, 18(2):192-205, March 1994.

8. E. Cuny, D. Guehl, P. Burbard, C. Gross, V. Dousset, and A. Rogier. Lack of agreement between direct magnetic resonance imaging and statistical determination of a subthalamic target: the role of electrophysiological guidance. Journal of Neurosurgery, pages 591-597, 2002.

9. P.F. D'Haese, E. Cetinkaya, P.E. Konrad, C. Kao, and B.M. Dawant. Computeraided placement of deep brain stimulators: From planning to intraoperative guidance. IEEE Transactions on Medical Imaging, 24(11).

10. P.F. D'Haese, S. Pallavaram, K. Niermann, J. Spooner, C. Kao, P.E. Konrad, and B.M. Dawant. Automatic selection of DBS target points using multiple electrophysiological atlases. In Seventh International Conference on Medical Image Computing and Computer Assisted Intervention MICCAI 2005, volume 2 of Lecture Notes in Computer Science, pages 427-434, Palm Springs, USA, October 2005. Springer.

11. E.G. Duerden, K.W. Finnis, T.M. Peters, and A.F. Sadikot. A method for analysis of electrophysiological responses obtained from the motor fibers of the human internal capsule. In Fifth International Conference on Medical Image Computing and Computer Assisted Intervention MICCAI 2003, Lecture Notes in Computer Science, pages 50-57, Montreal, Canada, November 2003. Springer. 
12. K.W. Finnis, Y.P. Starrveld, A.G. Parrent, A.F. Sadikot, and T.M. Peters. Threedimensional database of subcortical electrophysiology for image-guided stereotactic functional neurosurgery. IEEE Transactions on Medical Imaging, 22(1):93-104, January 2003.

13. T. Guo, K.W. Finnis, A.G. Parrent, and T.M. Peters. Development and application of functional databases for planning deep-brain neurosurgical procedures. In Seventh International Conference on Medical Image Computing and Computer Assisted Intervention MICCAI 2005, volume 1 of Lecture Notes in Computer Science, pages 835-842, Palm Springs, USA, October 2005. Springer.

14. C.J. Holmes, R. Hoge, L. Collins, R. Woods, A.W. Toga, and A.C. Evans. Enhancement of MR images using registration for signal averaging. Journal of Computer Assisted Tomography, 22(2):324-333, 1998.

15. M. Krause, W. Fogel, W. Hacke, M. Bonsanto, C. Trenkwalder, and V. Tronnier. Deep brain stimulation for the treatment of parkinson's disease: subthalamic nucleus versus globus pallidus internis. Journal of Neurology, Neurosurgery, and Psychiatry, 70:464-470, 2001.

16. W.L. Nowinski, D. Belov, and A.L. Benabid. An algorithm for rapid calcualation of a probablisitic functional atlas subcortical structures from electrophyisiological data collected during functional neurosurgical procedures. NeuroImage, 18:143$155,2003$.

17. S. Robbins, A.C. Evans, D.L. Collins, and S. Whitesides. Tuning and Comparing Spatial Normalization Methods. Medical Image Analysis, 8(3):311-323, 2004.

18. G. Schaltenbrand and W. Wahren. Atlas for Stereotaxy of the Human Brain. Georg Thieme Verlag, Stuttgart, Germany, 1977.

19. P. St-Jean, A.F. Sadikot, L. Collins, D. Clonda, R. Kasrai, A.C. Evans, and T.M. Peters. Automated Atlas Integration and Interactive Three-Dimensional Visualization Tools for Planning and Guidance in Functional Neurosurgery. IEEE Transactions on Medical Imaging (TMI), 17(5):854-866, May 1998.

20. P.A. Starr, J.L. Vitek, M. DeLong, and R.A. Bakay. Magnetic resonance imagingbased steretactic localization of the globus pallidus and subthalamic nucleus. Neurosurgery, 44(2):303-314, 1999.

21. J. Talairach and P. Tournoux. Co-Planar Stereotaxic Atlas of the Human Brain. Georg Thieme Verlag, Stuttgart, Germany, 1988. 